\title{
On the Structure of Compatible Rational Functions
}

\author{
Shaoshi Chen ${ }^{1,2,3}$, Ruyong Feng ${ }^{1}$, Guofeng $\mathrm{Fu}^{1}$, Ziming $\mathrm{Li}^{1}$ \\ ${ }^{1}$ Key Lab of Math.-Mech. AMSS, Chinese Academy of Sciences, Beijing 100190, (China) \\ ${ }_{2}^{2}$ Algorithms Project-Team, INRIA, Paris-Rocquencourt, 78513 Le Chesnay, (France) \\ ${ }^{3}$ RISC, Johannes Kepler University, 4040 Linz, (Austria) \\ \{schen, ryfeng\}@amss.ac.cn, \{fuguofeng, zmli\}@mmrc.iss.ac.cn
}

\begin{abstract}
A finite number of rational functions are compatible if they satisfy the compatibility conditions of a first-order linear functional system involving differential, shift and $q$-shift operators. We present a theorem that describes the structure of compatible rational functions. The theorem enables us to decompose a solution of such a system as a product of a rational function, several symbolic powers, a hyperexponential function, a hypergeometric term, and a $q$-hypergeometric term. We outline an algorithm for computing this product, and present an application.
\end{abstract}

\section{Categories and Subject Descriptors}

I.1.2 [Computing Methodologies]: Symbolic and Algebraic Manipulation-Algebraic Algorithms

\section{General Terms}

Algorithms, Theory

\section{Keywords}

Compatibility conditions, compatible rational functions, hyperexponential function, $(q-)$ hypergeometric term

\section{INTRODUCTION}

A linear functional system consists of linear partial differential, shift and $q$-shift operators. The commutativity of these operators implies that the coefficients of a linear functional system satisfy compatibility conditions.

\footnotetext{
*This work was supported in part by two grants of NSFC No. 60821002/F02 and No. 10901156. The first author was a PhD student in the Chinese Academy of Sciences and INRIA, Paris-Rocquencourt when the first draft of this paper was written. He is now a post doctoral fellow at RISC-Linz, and acknowledges the financial support by Austrian FWF grant Y464-N18
}

Permission to make digital or hard copies of all or part of this work for personal or classroom use is granted without fee provided that copies are not made or distributed for profit or commercial advantage and that copies bear this notice and the full citation on the first page. To copy otherwise, to republish, to post on servers or to redistribute to lists, requires prior specific permission and/or a fee.

ISSAC'11, June 8-11, 2011, San Jose, California, USA.

Copyright 2011 ACM 978-1-4503-0675-1/11/06 ...\$10.00.
A nonzero solution of a first-order linear partial differential system in one unknown function is called a hyperexponential function. Christopher and Zoladek [9, 21] use the compatibility (integrability) conditions to show that a hyperexponential function can be written as a product of a rational function, finitely many power functions, and an exponential function. Their results generalize a well-known fact, namely, for a rational function $r(t)$,

$$
\exp \left(\int r(t) d t\right)=f(t) r_{1}(t)^{e_{1}} \cdots r_{m}(t)^{e_{m}} \exp (g(t)),
$$

where $e_{1}, \ldots, e_{m}$ are constants, and $f, r_{1}, \ldots, r_{m}, g$ are rational functions. The generalization is useful to compute Liouvilian first integrals.

A nonzero solution of a first-order linear partial difference system in one unknown term is called a hypergeometric term. The Ore-Sato Theorem [16, 18] states that a hypergeometric term is a product of a rational function, several power functions and factorial terms. A $q$-analogue of the Ore-Sato theorem is given in $[11,8]$. All these results are based on compatibility conditions. The Ore-Sato theorem was rediscovered in one way or another, and is important for the proofs of a conjecture of Wilf and Zeilberger about holonomic hypergeometric terms $[2,4,17]$. This theorem and its $q$-analogue also play a crucial role in deriving criteria on the existence of telescopers for hypergeometric and $q$-hypergeometric terms, respectively $[1,8]$.

Consider a first-order mixed system

$$
\left\{\frac{\partial z(t, x)}{\partial t}=u(t, x) z(t, x), z(t, x+1)=v(t, x) z(t, x)\right\},
$$

where $u$ and $v$ are rational functions with $v \neq 0$. Its compatibility condition is $\partial v(t, x) / \partial t=v(t, x)(u(t, x+1)-u(t, x))$. By Proposition 5 in [10], a nonzero solution of the above system can be written as a product $f(t, x) r(t)^{x} \mathcal{E}(t) \mathcal{G}(x)$, where $f$ is a bivariate rational function in $t$ and $x, r$ is a univariate rational function in $t, \mathcal{E}$ is a hyperexponential function in $t$, and $\mathcal{G}$ is a hypergeometric term in $x$. This proposition is used to compute Liouvillian solutions of difference-differential systems.

In fact, the above proposition is also fundamental for the criteria on the existence of telescopers when both differential and shift operators are involved [7]. This motivates us to generalize the proposition to include differential, difference and $q$-difference cases. Such a generalization will enable us to establish the existence of telescopers when both differential (shift) and $q$-shift operators appear. Next, the proof of the Wilf-Zeilberger conjecture for hypergeometric terms is 
based on the Ore-Sato theorem. So it is reasonable to expect that a structural theorem on compatible rational functions with respect to differential, shift and $q$-shift operators helps us study the conjecture in more general cases.

The main result of this paper is Theorem 5.4 which reveals a special structure of compatible rational functions. By the theorem, a hyperexponential-hypergeometric solution, defined in Section 2, is a product of a rational function, several symbolic powers, a hyperexponential function, a hypergeometric term, and a $q$-hypergeometric term (see Proposition 6.1). This paves the way to decompose such solutions by Christopher-Zoladek's generalization, the OreSato Theorem, and its $q$-analogue.

This paper is organized as follows. The notion of compatible rational functions is introduced in Section 2. The bivariate case is studied in Section 3. After presenting a few preparation lemmas in Section 4, we prove in Section 5 a theorem that describes the structure of compatible rational functions. Section 6 is about algorithms and applications.

\section{COMPATIBLE RATIONAL FUNCTIONS}

In the rest of this paper, $\mathbb{F}$ is a field of characteristic zero. Let $\mathbf{t}=\left(t_{1}, \ldots, t_{l}\right), \mathbf{x}=\left(x_{1}, \ldots, x_{m}\right)$ and $\mathbf{y}=\left(y_{1}, \ldots, y_{n}\right)$. Assume that $q_{1}, \ldots, q_{n} \in \mathbb{F}$ are neither zero nor roots of unity. For an element $f$ of $\mathbb{F}(\mathbf{t}, \mathbf{x}, \mathbf{y})$, define $\delta_{i}(f)=\partial f / \partial t_{i}$ for all $i$ with $1 \leq i \leq l$,

$\sigma_{j}(f(\mathbf{t}, \mathbf{x}, \mathbf{y}))=f\left(\mathbf{t}, x_{1}, \ldots, x_{j-1}, x_{j}+1, x_{j+1}, \ldots, x_{m}, \mathbf{y}\right)$

for all $j$ with $1 \leq j \leq m$, and

$$
\tau_{k}(f(\mathbf{t}, \mathbf{x}, \mathbf{y}))=f\left(\mathbf{t}, \mathbf{x}, y_{1}, \ldots, y_{k-1}, q_{k} y_{k}, y_{k+1}, \ldots, y_{n}\right)
$$

for all $k$ with $1 \leq k \leq n$. They are called derivations, shift operators, and $q$-shift operators, respectively.

Let $\Delta=\left\{\delta_{1}, \ldots, \delta_{l}, \sigma_{1}, \ldots, \sigma_{m}, \tau_{1}, \ldots, \tau_{n}\right\}$. These operators commute pairwise. The field of constants w.r.t. an operator in $\Delta$ consists of all rational functions free of the indeterminate on which the operator acts nontrivially.

By a first-order linear functional system over $\mathbb{F}(\mathbf{t}, \mathbf{x}, \mathbf{y})$, we mean a system consisting of

$$
\delta_{i}(z)=u_{i} z, \sigma_{j}(z)=v_{j} z, \tau_{k}(z)=w_{k} z
$$

for some rational functions $u_{i}, v_{j}, w_{k} \in \mathbb{F}(\mathbf{t}, \mathbf{x}, \mathbf{y})$ and for all $i, j, k$ with $1 \leq i \leq l, 1 \leq j \leq m$ and $1 \leq k \leq n$. System (1) is said to be compatible if

$$
v_{1} \cdots v_{m} w_{1} \cdots w_{n} \neq 0
$$

and the conditions listed in (3)-(8) hold:

$$
\begin{gathered}
\delta_{i}\left(u_{j}\right)=\delta_{j}\left(u_{i}\right), \quad 1 \leq i<j \leq l, \\
\sigma_{i}\left(v_{j}\right) / v_{j}=\sigma_{j}\left(v_{i}\right) / v_{i}, \quad 1 \leq i<j \leq m, \\
\tau_{i}\left(w_{j}\right) / w_{j}=\tau_{j}\left(w_{i}\right) / w_{i}, \quad 1 \leq i<j \leq n, \\
\delta_{i}\left(v_{j}\right) / v_{j}=\sigma_{j}\left(u_{i}\right)-u_{i}, \quad 1 \leq i \leq l \text { and } 1 \leq j \leq m, \\
\delta_{i}\left(w_{k}\right) / w_{k}=\tau_{k}\left(u_{i}\right)-u_{i}, \quad 1 \leq i \leq l \text { and } 1 \leq k \leq n, \\
\sigma_{j}\left(w_{k}\right) / w_{k}=\tau_{k}\left(v_{j}\right) / v_{j}, \quad 1 \leq j \leq m \text { and } 1 \leq k \leq n .
\end{gathered}
$$

Compatibility conditions (3)-(8) are caused by the commutativity of the maps in $\Delta$. A sequence of rational functions: $u_{1}, \ldots, u_{l}, v_{1}, \ldots, v_{m}, w_{1}, \ldots, w_{n}$ is said to be compatible w.r.t. $\Delta$ if (2)-(8) hold.
By a $\Delta$-extension of $\mathbb{F}(\mathbf{t}, \mathbf{x}, \mathbf{y})$, we mean a ring extension $R$ of $\mathbb{F}(\mathbf{t}, \mathbf{x}, \mathbf{y})$ s.t. every derivation and automorphism in $\Delta$ can be extended to a derivation and a monomorphism from $R$ to $R$, and, moreover, the extended maps are commutative with each other. Given a finite number of first-order compatible systems, one can construct a Picard-Vessiot $\Delta$ extension of $\mathbb{F}(\mathbf{t}, \mathbf{x}, \mathbf{y})$ that contains "all" solutions of these systems. Moreover, every nonzero solution is invertible. Details on Picard-Vessiot extensions of compatible systems may be found in [5]. More general and powerful extensions are described in [12]. By a hyperexponential-hypergeometric solution $h$ over $\mathbb{F}(\mathbf{t}, \mathbf{x}, \mathbf{y})$, we mean a nonzero solution of the system (1). The coefficients $u_{i}, v_{j}$ and $w_{k}$ in (1) are called

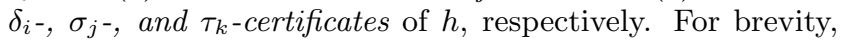
we abbreviate "hyperexponential-hypergeometric solution" as " $H$-solution". An $H$-solution is a hyperexponential function when $m=n=0$ in (1), it is a hypergeometric term if $l=n=0$, and a $q$-hypergeometric term if $l=m=0$.

REMARK 2.1. We opt for the word "solution" rather than "function", since all the $t_{i}, x_{j}$ and $y_{k}$ are regarded as indeterminates. It is more sophisticated to regard hypergeometric terms as functions of integer variables [17, 4, 3].

As a matter of notation, for an element $f \in \mathbb{F}(\mathbf{t}, \mathbf{x}, \mathbf{y})$, the denominator and numerator of $f$ are denoted $\operatorname{den}(f)$ and $\operatorname{num}(f)$, respectively. Note that $\operatorname{den}(f)$ and $\operatorname{num}(f)$ are coprime. For a ring $\mathbb{A}, \mathbb{A}^{\times}$stands for $\mathbb{A} \backslash\{0\}$, and for a field $\mathbb{E}, \overline{\mathbb{E}}$ stands for the algebraic closure of $\mathbb{E}$. For every $\phi \in \Delta$ and $f \in \mathbb{F}(\mathbf{t}, \mathbf{x}, \mathbf{y})^{\times}$, we denote by $\ell \phi(f)$ the fraction $\phi(f) / f$. When $\phi$ is a derivation $\delta_{i}, \ell \delta_{i}(f)$ stands for the logarithmic derivative of $f$ with respect to $t_{i}$. This notation allows us to avoid stacking fractions and subscripts.

Let $\mathbb{E}$ be a field and $t$ an indeterminate. A nonzero element $f$ of $\mathbb{E}(t)$ can be written uniquely as $f=p+r$, where $p \in \mathbb{E}[t]$ and $r$ is a proper fraction. We say that $p$ is the polynomial part of $f$ w.r.t. $t$.

REMARK 2.2. Let $z \in\left\{t_{1}, \ldots, t_{l}, x_{1}, \ldots, x_{m}, y_{1}, \ldots, y_{n}\right\}$ and $f \in \mathbb{F}(\mathbf{t}, \mathbf{x}, \mathbf{y})^{\times}$. For all $i$ with $1 \leq i \leq l$, the polynomial part of $\ell \delta_{i}(f)$ w.r.t. $z$ has degree at most zero in $z$.

\section{BIVARIATE CASE}

In this section, we assume that $l=m=n=1$. For brevity, set $t=t_{1}, x=x_{1}, y=y_{1}, \delta=\delta_{1}, \sigma=\sigma_{1}, \tau=\tau_{1}$, and $q=q_{1}$. By (2), (6), (7) and (8), three rational functions $u, v, w$ in $\mathbb{F}(t, x, y)$ are $\Delta$-compatible if $v w \neq 0$,

$$
\begin{gathered}
\ell \delta(v)=\sigma(u)-u, \\
\ell \delta(w)=\tau(u)-u, \\
\ell \sigma(w)=\ell \tau(v) .
\end{gathered}
$$

Other compatibility conditions become trivial in this case.

Example 3.1. Let $\alpha \in \mathbb{F}(t, y)^{\times}$. The system consisting of $\delta(z)=\ell \delta(\alpha) x z$ and $\sigma(z)=\alpha z$ is compatible w.r.t. $\delta$ and $\sigma$. Denote a solution of this system by $\alpha^{x}$, which is irrational if $\alpha \neq 1$.

The next lemma is immediate from [10, Proposition 5].

Lemma 3.1. Let $u, v \in \mathbb{F}(t, x, y)$ with $v \neq 0$. If (9) holds, then $u=\ell \delta(f)+\ell \delta(\alpha) x+\beta$ and $v=\ell \sigma(f) \alpha \lambda$ for some $f$ in $\mathbb{F}(t, x, y), \alpha, \beta$ in $\mathbb{F}(t, y)$, and $\lambda$ in $\mathbb{F}(x, y)$. 
Assume that an $H$-solution $h$ has $\delta$-certificate $u$ and $\sigma$ certificate $v$. By Lemma 3.1, $h=c f \alpha^{x} \mathcal{E} \mathcal{G}$ in some $\Delta$-ring, where $c$ is a constant w.r.t. $\delta$ and $\sigma, \mathcal{E}$ is hyperexponential with certificate $\beta$, and $\mathcal{G}$ is hypergeometric with certificate $\lambda$.

We shall prove two similar results: one is about differential and $q$-shift variables; the other about shift and $q$-shift ones. To this end, we recall some terminologies from $[2,4,12]$.

Let $\mathbb{A}=\mathbb{F}(t, y)$ and $p \in \mathbb{A}[x]^{\times}$. The $\sigma$-dispersion of $p$ is defined to be the largest nonnegative integer $i$ s.t. for some $r$ in $\overline{\mathbb{A}}, r$ and $r+i$ are roots of $p$. Let $f \in \mathbb{A}(x)^{\times}$. We say that $f$ is $\sigma$-reduced if $\operatorname{den}(f)$ and $\sigma^{i}(\operatorname{num}(f))$ are coprime for every integer $i$; and that $f$ is $\sigma$-standard if zero is the $\sigma$ dispersion of num $(f) \operatorname{den}(f)$. A $\sigma$-standard rational function is a $\sigma$-reduced one, but the converse is false. By Lemma 6.2 in [12], $f=\ell \sigma(a) b$ for some $a, b$ in $\mathbb{A}(x)$ with $b$ being $\sigma$ standard or $\sigma$-reduced.

Let $\mathbb{B}=\mathbb{F}(t, x)$ and $p \in \mathbb{B}[y]^{\times}$. The $\tau$-dispersion of $p$ is defined to be the largest nonnegative integer $i$ s.t. for some nonzero $r \in \overline{\mathbb{B}}, r$ and $q^{i} r$ are roots of $p$. In addition, the $\tau$-dispersion of $p$ is set to be zero if $p=c y^{k}$ for some $c \in \mathbb{B}$. Let $f \in \mathbb{B}(y)^{\times}$. The polar $\tau$-dispersion is the $\tau$-dispersion of $\operatorname{den}(f)$. The notion of $\tau$-reduced and $\tau$-standard rational functions are defined likewise. One can write $f=\ell \tau(a) b$, where $a, b \in \mathbb{B}(y)^{\times}$and $b$ is $\tau$-standard or $\tau$-reduced.

Now, we prove a $q$-analogue of Lemma 3.1.

Lemma 3.2. Let $u, w \in \mathbb{F}(t, x, y)$ with $w \neq 0$. If (10) holds, then $u=\ell \delta(f)+a$ and $w=\ell \tau(f) b$ for some $f$ in $\mathbb{F}(t, x, y)$, $a$ in $\mathbb{F}(t, x)$, and $b$ in $\mathbb{F}(x, y)$.

Proof. Set $w=\ell \tau(f) b$ for some $f, b$ in $\mathbb{F}(t, x, y)$ with $b$ being $\tau$-standard. Set $b=y^{k} P / Q$, where $P, Q \in \mathbb{F}(x)[t, y]$ are coprime, and neither is divisible by $y$. Since $b$ is $\tau$ standard, so is $P / Q$. Assume $u=\ell \delta(f)+a$. By (10),

$$
\ell \delta(P / Q)=\tau(a)-a .
$$

Since $P / Q$ is $\tau$-standard, the $\tau$-dispersion of $P Q$ is zero, and so is the polar $\tau$-dispersion of the left-hand side in (12), which, together with [12, Lemma 6.3], implies that $a$ belongs to $\mathbb{F}(t, x)\left[y, y^{-1}\right]$. Moreover, $a$ is free of positive powers of $y$ by Remark 2.2 (setting $z=y$ ); and $a$ is free of negative powers of $y$, because neither $P$ nor $Q$ is divisible by $y$. We conclude that $a$ is in $\mathbb{F}(t, x)$. Consequently, $\tau(a)=a$. It follows from (12) that $\delta(P / Q)=0$, i.e., $b$ is in $\mathbb{F}(x, y)$.

By the above lemma, an $H$-solution $h$ can be written as a product of a constant w.r.t. $\delta$ and $\tau$, a rational function, a hyperexponential function, and a $q$-hypergeometric term.

The last lemma is a $q$-analogue of [4, Theorem 9]. Our proof is based on an easy consequence of [20, Lemma 2.1].

FACT 3.1. Let $a, b \in \mathbb{F}(t, x, y)^{\times}$. If $\sigma(a)=b a$, and $P$ is an irreducible factor of $\operatorname{den}(b)$ with $\operatorname{deg}_{x} P>0$, then $\sigma^{i}(P)$ is a factor of num(b) for some nonzero integer $i$.

The same is true if we swap $\operatorname{den}(b)$ and $\operatorname{num}(b)$ in the above assertion.

Lemma 3.3. Let $v, w \in \mathbb{F}(t, x, y)^{\times}$. If (11) holds, then $v=\ell \sigma(f) a$ and $w=\ell \tau(f) b$ for some $f$ in $\mathbb{F}(t, x, y)$, a in $\mathbb{F}(t, x)$, and $b$ in $\mathbb{F}(t, y)$.

Proof. In this proof, $P \mid Q$ means that $P, Q \in \mathbb{F}(t)[x, y]^{\times}$ and $Q=P R$ for some $R \in \mathbb{F}(t)[x, y]$.

Set $v=\ell \sigma(f) a$, where $f, a \in \mathbb{F}(t, x, y)$ and $a$ is $\sigma$-reduced. Assume $w=\ell \tau(f) b$. By (11), $\ell \sigma(b)=\ell \tau(a)$, that is,

$$
\sigma(b)=g b, \quad \text { where } g=\frac{\tau(\operatorname{num}(a)) \operatorname{den}(a)}{\tau(\operatorname{den}(a)) \operatorname{num}(a)} .
$$

First, we show that $a$ is the product of an element in $\mathbb{F}(t, x)$ and an element in $\mathbb{F}(t, y)$. Suppose the contrary. Then there is an irreducible polynomial $P \in \mathbb{F}(t)[x, y]$ with $\operatorname{deg}_{x} P>0$ and $\operatorname{deg}_{y} P>0$ s.t. $P$ divides $\operatorname{den}(a) \operatorname{num}(a)$ in $\mathbb{F}(t)[x, y]$. Assume that $P \mid \operatorname{num}(a)$. If $P \nmid \operatorname{den}(g)$, then $P \mid \tau(\operatorname{num}(a))$ since $\operatorname{num}(a)$ and $\operatorname{den}(a)$ are coprime. So $\tau^{-1}(P) \mid \operatorname{num}(a)$. If $P \mid \operatorname{den}(g)$, then $\sigma^{i}(P) \mid \operatorname{num}(g)$ for some integer $i$ by (13) and Fact 3.1. Thus, $\sigma^{i}(P) \mid \tau(\operatorname{num}(a))$, because $\operatorname{num}(g)$ is a factor of $\tau(\operatorname{num}(a)) \operatorname{den}(a)$ and $a$ is $\sigma$-reduced. This implies $\sigma^{i} \tau^{-1}(P) \mid \operatorname{num}(a)$. In either case, we have that

$$
\sigma^{j} \tau^{-1}(P) \mid \operatorname{num}(a) \text { for some integer } j .
$$

Assume $P \mid \operatorname{den}(a)$. Then the same argument implies

$$
\sigma^{k} \tau^{-1}(P) \mid \operatorname{den}(a) \text { for some integer } k \text {. }
$$

Hence, there exists an integer $m_{1}$ s.t. $P_{1}:=\sigma^{m_{1}} \tau^{-1}\left(P_{0}\right)$ is an irreducible factor of $\operatorname{den}(a) \operatorname{num}(a)$, where $P_{0}=P$. A repeated use of the above reasoning leads to an infinite sequence of irreducible polynomials $P_{0}, P_{1}, P_{2}, \ldots$ in $\mathbb{F}(t)[x, y]$ s.t. $P_{i}=\sigma^{m_{i}} \tau^{-1}\left(P_{i-1}\right)$ and $P_{i} \mid \operatorname{den}(a) \operatorname{num}(a)$. Therefore, there are two $\mathbb{F}(t)$-linearly dependent members in the sequence. Using these two members, we get $P_{0}=c \sigma^{m} \tau^{n}\left(P_{0}\right)$ for some $c$ in $\mathbb{F}(t)$ and $m, n$ in $\mathbb{Z}$ with $n \neq 0$. Write

$$
P_{0}=p_{d}(x) y^{d}+p_{d-1}(x) y^{d-1}+\cdots+p_{0}(x),
$$

where $d>0, p_{i} \in \mathbb{F}(t)[x]$ and $p_{d} \neq 0$. Then

$$
p_{d}(x)=c p_{d}(x+m) q^{-d n} \quad \text { and } \quad p_{0}(x)=c p_{0}(x+m) .
$$

Since $P_{0}$ is irreducible and of positive degree in $x, p_{0}$ is also nonzero. We see that $1=c q^{-d n}$ and $1=c$ when comparing the leading coefficients in the above two equalities. Consequently, $q$ is a root of unity, a contradiction. This proves that all irreducible factors of $\operatorname{den}(a) \operatorname{num}(a)$ are either in $\mathbb{F}(t)[x]$ or $\mathbb{F}(t)[y]$. Therefore, $a$ is a product of an element in $\mathbb{F}(t, x)$ and an element in $\mathbb{F}(t, y)$.

So we can write $a=a_{1} a_{2}$ for some $a_{1}$ in $\mathbb{F}(t, x)$ and $a_{2}$ in $\mathbb{F}(t, y)$. By $\ell \sigma(b)=\ell \tau(a)$, the equation $\sigma(z)=\ell \tau\left(a_{2}\right) z$ has a rational solution $b$. Since $\ell \tau\left(a_{2}\right)$ is a constant w.r.t. $\sigma$, we conclude $\ell \tau\left(a_{2}\right)=1$, for otherwise, $\sigma(z)=\ell \tau\left(a_{2}\right) z$ would have no rational solution. So $b \in \mathbb{F}(t, y)$ and $a \in \mathbb{F}(t, x)$.

Similar to Lemmas 3.1 and 3.2, the above lemma implies that an $H$-solution $h$ can be written as a product of a constant w.r.t. $\sigma$ and $\tau$, a rational function, a hypergeometric term, and a $q$-hypergeometric term.

We shall extend these lemmas to multivariate cases in Section 5. Before closing this section, we present three examples to illustrate calculations involving compatibility conditions. These calculations are useful in Section 5.

\section{ExAmple 3.2. Assume}

$$
u=\ell \delta(f)+\ell \delta(a) x+b \quad \text { and } \quad v=\ell \sigma(f) a c,
$$

where $f, c \in \mathbb{F}(t, x, y)^{\times}, a \in \mathbb{F}(t, y)^{\times}$, and $b \in \mathbb{F}(t, x, y)$. By the logarithmic derivative identity: for all $r, s$ in $\mathbb{F}(t, x, y)^{\times}$, $\ell \delta(r s)=\ell \delta(r)+\ell \delta(s)$, we get

$$
\ell \delta(v)=\ell \delta \circ \ell \sigma(f)+\ell \delta(a)+\ell \delta(c) .
$$

Since $\ell \delta(a)$ is constant w.r.t. $\sigma$, and $\sigma \circ \ell \delta=\ell \delta \circ \sigma$, we have

$$
\begin{aligned}
\sigma(u)-u & =\sigma \circ \ell \delta(f)-\ell \delta(f)+\ell \delta(a)+\sigma(b)-b \\
& =\ell \delta \circ \ell \sigma(f)+\ell \delta(a)+\sigma(b)-b .
\end{aligned}
$$


If (9) holds, then $\ell \delta(c)=\sigma(b)-b$. Hence, $\delta(c)=0$ iff $\sigma(b)=b$, i.e., $c \in \mathbb{F}(x, y)$ iff $b \in \mathbb{F}(t, y)$.

ExAmple 3.3. Assume $u=\ell \delta(f)+a$ and $w=\ell \tau(f) b$, where $a \in \mathbb{F}(t, x, y)$ and $f, b \in \mathbb{F}(t, x, y)^{\times}$. If (10) holds, then a similar calculation as above yields $\ell \delta(b)=\tau(a)-a$. Hence, $\delta(b)=0$ iff $\tau(a)=a$, i.e., $b \in \mathbb{F}(x, y)$ iff $a \in \mathbb{F}(t, x)$.

ExAmPle 3.4. Assume $v=\ell \sigma(f) a$ and $w=\ell \tau(f) b$, where $f, a, b \in \mathbb{F}(t, x, y)^{\times}$. Applying $\ell \sigma, \ell \tau$ to $w, v$, respectively, we see that

$$
\ell \sigma(w)=\ell \sigma \circ \ell \tau(f) \ell \sigma(b), \quad \ell \tau(v)=\ell \tau \circ \ell \sigma(f) \ell \tau(a) .
$$

If (11) holds, then $\ell \sigma(b)=\ell \tau(a)$, because $\ell \sigma \circ \ell \tau=\ell \tau \circ \ell \sigma$. Hence, $\sigma(b)=b$ iff $\tau(a)=a$, i.e., $b \in \mathbb{F}(t, y)$ iff $a \in \mathbb{F}(t, x)$.

\section{PREPARATION LEMMAS}

To extend Lemmas 3.1, 3.2, and 3.3 to multivariate cases, we will proceed by induction on the number of variables. There arise different expressions for a rational function in our induction. Lemmas given in this section will be used to eliminate redundant indeterminates in these expressions.

We define a few additive subgroups of $\mathbb{F}(\mathbf{t}, \mathbf{x}, \mathbf{y})$ to avoid complicated expressions.

$$
\begin{gathered}
L_{i}=\left\{\ell \delta_{i}(f) \mid f \in \mathbb{F}(\mathbf{t}, \mathbf{x}, \mathbf{y})^{\times}\right\}, i=1, \ldots, l, \\
M_{i}=\left\{\sum_{j=1}^{m} \ell \delta_{i}\left(g_{j}\right) x_{j} \mid g_{j} \in \mathbb{F}(\mathbf{t}, \mathbf{y})^{\times}\right\}, i=1, \ldots, l .
\end{gathered}
$$

For $i=1, \ldots, l$ and $j=1, \ldots, m, M_{i, j}$ denotes the group

$$
\left\{\sum_{k=1}^{j-1} \ell \delta_{i}\left(g_{k}\right) x_{k}+\sum_{k=j+1}^{m} \ell \delta_{i}\left(g_{k}\right) x_{k} \mid g_{k} \in \mathbb{F}\left(\mathbf{t}, x_{j}, \mathbf{y}\right)^{\times}\right\} \text {. }
$$

Moreover, we set

$N_{i}=L_{i}+M_{i}+\mathbb{F}(\mathbf{t}, \mathbf{y}) \quad$ and $\quad N_{i, j}=L_{i}+M_{i, j}+\mathbb{F}\left(\mathbf{t}, x_{j}, \mathbf{y}\right)$.

Let $Z=\left\{t_{1}, \ldots, t_{l}, x_{1}, \ldots, x_{m}, y_{1}, \ldots, y_{n}\right\}$. We will use an evaluation trick in the sequel. Let $Z^{\prime}=\left\{z_{1}, \ldots, z_{s}\right\}$ be a subset of $Z$. For $f \in \mathbb{F}(\mathbf{t}, \mathbf{x}, \mathbf{y})^{\times}$, there exist $\xi_{1}, \ldots, \xi_{s}$ in $\mathbb{F}$ s.t. $f$ evaluated at $z_{1}=\xi_{1}, \ldots, z_{s}=\xi_{s}$ is a well-defined and nonzero rational function $f^{\prime}$. We say that $f^{\prime}$ is a proper evaluation of $f$ w.r.t. $Z^{\prime}$. A proper evaluation can be carried out for finitely many rational functions as well. In addition, we say that a rational function $f$ is free of $Z^{\prime}$ if it is free of every indeterminate in $Z^{\prime}$

REMARK 4.1. If $Z^{\prime} \subset Z, f \in L_{i}$ and $t_{i} \notin Z^{\prime}$, then all proper evaluations of $f$ w.r.t. $Z^{\prime}$ are also in $L_{i}$.

In the next example, we illustrate two typical proper evaluations to be used later.

EXAMPLE 4.2. Let $f=\ell \delta_{i}(r)$ for some $f, r \in \mathbb{F}(\mathbf{t}, \mathbf{x}, \mathbf{y})^{\times}$. Assume that both $f(\mathbf{t}, \boldsymbol{\xi}, \mathbf{y})$ and $r(\mathbf{t}, \boldsymbol{\xi}, \mathbf{y})$ are well-defined and nonzero, where $\boldsymbol{\xi} \in \mathbb{F}^{m}$. Then $f(\mathbf{t}, \boldsymbol{\xi}, \mathbf{y})$ is still in $L_{i}$.

Let $g \in \mathbb{F}(\mathbf{t}, \mathbf{y})^{\times}$. Then $\delta_{i}(z)=g z$ has a rational solution in $\mathbb{F}(\mathbf{t}, \mathbf{y})^{\times}$if it has a rational solution in $\mathbb{F}(\mathbf{t}, \mathbf{x}, \mathbf{y})^{\times}$. This can also be shown by a proper evaluation.

The following lemma helps us merge rational expressions involving logarithmic derivatives.
Lemma 4.1. Let $i \in\{1, \ldots, l\}$.

(i) Let $Z_{1}, Z_{2} \subset Z$ with $Z_{1} \cap Z_{2}=\emptyset$. If $\mathbb{A}$ is any subfield of $\mathbb{F}(\mathbf{t}, \mathbf{x}, \mathbf{y})$ whose elements are free of $t_{i}$ and free of $Z_{1} \cup Z_{2}$, then

$$
L_{i}+\mathbb{A}\left(t_{i}\right)=\left(L_{i}+\mathbb{A}\left(t_{i}, Z_{1}\right)\right) \cap\left(L_{i}+\mathbb{A}\left(t_{i}, Z_{2}\right)\right) .
$$

(ii) If $d, e \in\{1, \ldots, m\}$ with $d \neq e$, then $N_{i}=N_{i, d} \cap N_{i, e}$.

Proof. To prove the first assertion, note that $L_{i}+\mathbb{A}\left(t_{i}\right)$ is a subset of $\left(L_{i}+\mathbb{A}\left(t_{i}, Z_{1}\right)\right) \cap\left(L_{i}+\mathbb{A}\left(t_{i}, Z_{2}\right)\right)$. Assume that $a$ is in $\left(L_{i}+\mathbb{A}\left(t_{i}, Z_{1}\right)\right) \cap\left(L_{i}+\mathbb{A}\left(t_{i}, Z_{2}\right)\right)$. Then there exist $a_{1} \in \mathbb{A}\left(t_{i}, Z_{1}\right)$ and $a_{2} \in \mathbb{A}\left(t_{i}, Z_{2}\right)$ s.t.

$$
a \equiv a_{1} \quad \bmod L_{i} \quad \text { and } \quad a \equiv a_{2} \quad \bmod L_{i} .
$$

Hence, $a_{1}-a_{2} \in L_{i}$. Let $Z_{2}^{\prime}=Z_{2} \backslash\left\{t_{i}\right\}$, and $a_{2}^{\prime}$ be a proper evaluation of $a_{2}$ w.r.t. $Z_{2}^{\prime}$. Then $a_{1}-a_{2}^{\prime}$ is a proper evaluation of $a_{1}-a_{2}$ w.r.t. $Z_{2}^{\prime}$, because $a_{1}$ is free of $Z_{2}^{\prime}$. Thus, $a_{1}-a_{2}^{\prime}$ belongs to $L_{i}$ by Remark 4.1. Since $a_{2}^{\prime}$ is in $\mathbb{A}\left(t_{i}\right), a_{1}$ is in $L_{i}+\mathbb{A}\left(t_{i}\right)$, and so is $a$.

To prove the second assertion, assume $i=1, d=1$ and $e=m$. Note that $N_{1} \subset N_{1,1} \cap N_{1, m}$, because $M_{1}$ is contained in $\left(M_{1,1}+\mathbb{F}\left(\mathbf{t}, x_{1}, \mathbf{y}\right)\right) \cap\left(M_{1, m}+\mathbb{F}\left(\mathbf{t}, x_{m}, \mathbf{y}\right)\right)$. It remains to show $N_{1,1} \cap N_{1, m} \subset N_{1}$. Let $a \in N_{1,1} \cap N_{1, m}$. Then

$$
\begin{aligned}
a & =\ell \delta_{1}(f)+\left(\sum_{j=2}^{m-1} \ell \delta_{1}\left(g_{j}\right) x_{j}\right)+\ell \delta_{1}\left(g_{m}\right) x_{m}+r \\
& =\ell \delta_{1}(\tilde{f})+\ell \delta_{1}\left(\tilde{g}_{1}\right) x_{1}+\left(\sum_{j=2}^{m-1} \ell \delta_{1}\left(\tilde{g}_{j}\right) x_{j}\right)+\tilde{r}
\end{aligned}
$$

where $f, \tilde{f} \in \mathbb{F}(\mathbf{t}, \mathbf{x}, \mathbf{y}), g_{j}, r \in \mathbb{F}\left(\mathbf{t}, x_{1}, \mathbf{y}\right), \tilde{g}_{j}, \tilde{r} \in \mathbb{F}\left(\mathbf{t}, x_{m}, \mathbf{y}\right)$ and $f \tilde{f} g_{j} \tilde{g}_{j} \neq 0$. For all $j$ with $1 \leq j \leq m$, let $P_{j}$ be the polynomial part of $a$ w.r.t. $x_{j}$. Then $\operatorname{deg}_{x_{j}} P_{j} \leq 1$ for all $j$ with $1 \leq j \leq m-1$ by Remark 2.2 and (15), and $\operatorname{deg}_{x_{m}} P_{m} \leq 1$ by the same Remark and (14).

Claim. Let $b_{j}$ denote the coefficient of $x_{j}$ in $P_{j}$. Then there exists $s_{j} \in \mathbb{F}(\mathbf{t}, \mathbf{y})$ s.t. $b_{j}=\ell \delta_{1}\left(s_{j}\right)$ for all $j$ with $1 \leq j \leq m$. Proof of Claim. By (14) and Remark 2.2, $b_{1}$ is the coefficient of $x_{1}$ in the polynomial part of $r$ w.r.t. $x_{1}$. So $b_{1}$ is in $\mathbb{F}(\mathbf{t}, \mathbf{y})$. By (15) and the same remark, $b_{1}=\ell \delta_{1}\left(\tilde{g}_{1}\right)$. Let $s_{1}$ be a proper evaluation of $\tilde{g}_{1}$ w.r.t. $x_{m}$. Then $b_{1}=\ell \delta_{1}\left(s_{1}\right)$ as $b_{1}$ is free of $x_{m}$. By the same argument, $b_{m}=\ell \delta_{1}\left(s_{m}\right)$ for some $s_{m}$ in $\mathbb{F}(\mathbf{t}, \mathbf{x})$. By (14) and (15), $b_{j}=\ell \delta_{1}\left(g_{j}\right)=\ell \delta_{1}\left(\tilde{g}_{j}\right)$ for all $j$ with $2 \leq j \leq m-1$. Let $s_{j}$ be a proper evaluation of $\tilde{g}_{j}$ w.r.t. $x_{m}$. Then $\ell \delta_{j}\left(g_{j}\right)=\ell \delta_{j}\left(s_{j}\right)$, because $g_{j}$ is free of $x_{m}$. Hence, $b_{j}=\ell \delta_{1}\left(s_{j}\right)$. The claim holds.

Set $b=\sum_{j=1}^{m} b_{j} x_{j}$. Then $a-b$ is in $L_{1}+\mathbb{F}\left(\mathbf{t}, x_{1}, \mathbf{y}\right)$ and $L_{1}+\mathbb{F}\left(\mathbf{t}, x_{m}, \mathbf{y}\right)$ by (14), (15) and the claim. Thus, $a-b$ is in $L_{1}+\mathbb{F}(\mathbf{t}, \mathbf{y})$ by the first assertion (setting $Z_{1}=\left\{x_{1}\right\}$, $Z_{2}=\left\{x_{m}\right\}$, and $\left.\mathbb{A}=\mathbb{F}\left(t_{2}, \ldots, t_{l}, \mathbf{y}\right)\right)$. By the claim, $b$ is in $M_{1}$. Thus, $a$ is in $L_{1}+M_{1}+\mathbb{F}(\mathbf{t}, \mathbf{y})$.

We define a few multiplicative subgroups in $\mathbb{F}(\mathbf{t}, \mathbf{x}, \mathbf{y})^{\times}$. Let $G_{j}=\left\{\ell \sigma_{j}(f) \mid f \in \mathbb{F}(\mathbf{t}, \mathbf{x}, \mathbf{y})^{\times}\right\}$for $j=1, \ldots, m$. Similarly, let $H_{k}=\left\{\ell \tau_{k}(f) \mid f \in \mathbb{F}(\mathbf{t}, \mathbf{x}, \mathbf{y})^{\times}\right\}$for $k=1, \ldots, n$.

REMARK 4.3. If $Z^{\prime} \subset Z, f \in H_{k}$ and $y_{k} \notin Z^{\prime}$, then all proper evaluations of $f$ w.r.t. $Z^{\prime}$ are in $H_{k}$. The same holds for $G_{j}$.

The next lemma helps us merge rational expressions involving shift or $q$-shift quotients.

Lemma 4.2. Let $j \in\{1, \ldots, m\}, k \in\{1, \ldots, n\}$. Assume that $Z_{1}$ and $Z_{2}$ are disjoint subsets of $Z$. 
(i) If $\mathbb{A}$ is any subfield of $\mathbb{F}(\mathbf{t}, \mathbf{x}, \mathbf{y})$ whose elements are free of $x_{j}$ and free of $Z_{1} \cup Z_{2}$, then

$$
G_{j} \mathbb{A}\left(x_{j}\right)^{\times}=\left(G_{j} \mathbb{A}\left(x_{j}, Z_{1}\right)^{\times}\right) \cap\left(G_{j} \mathbb{A}\left(x_{j}, Z_{2}\right)^{\times}\right) .
$$

(ii) If $\mathbb{A}$ is any subfield of $\mathbb{F}(\mathbf{t}, \mathbf{x}, \mathbf{y})$ whose elements are free of $y_{k}$ and free of $Z_{1} \cup Z_{2}$, then

$$
H_{k} \mathbb{A}\left(y_{k}\right)^{\times}=\left(H_{k} \mathbb{A}\left(y_{k}, Z_{1}\right)^{\times}\right) \cap\left(H_{k} \mathbb{A}\left(y_{k}, Z_{2}\right)^{\times}\right) .
$$

(iii) If $\mathbb{A}=\mathbb{F}(\mathbf{t}, \mathbf{y})$ and $\mathbb{B}=\mathbb{F}(\mathbf{x}, \mathbf{y})$, then

$$
G_{j} \mathbb{A}^{\times} \mathbb{B}^{\times}=\left(G_{j} \mathbb{A}^{\times} \mathbb{B}\left(Z_{1}\right)^{\times}\right) \cap\left(G_{j} \mathbb{A}^{\times} \mathbb{B}\left(Z_{2}\right)^{\times}\right) .
$$

Proof. The proofs of the first two assertions are similar to that of Lemma 4.1 (i). So we only outline the proof of the second assertion. Clearly,

$$
H_{k} \mathbb{A}\left(y_{k}\right)^{\times} \subset\left(H_{k} \mathbb{A}\left(y_{k}, Z_{1}\right)^{\times}\right) \cap\left(H_{k} \mathbb{A}\left(y_{k}, Z_{2}\right)^{\times}\right) .
$$

For an element $a \in\left(H_{k} \mathbb{A}\left(y_{k}, Z_{1}\right)^{\times}\right) \cap\left(H_{k} \mathbb{A}\left(y_{k}, Z_{2}\right)^{\times}\right)$, there exist $a_{1} \in \mathbb{A}\left(y_{k}, Z_{1}\right)^{\times}$and $a_{2} \in \mathbb{A}\left(y_{k}, Z_{2}\right)^{\times}$s.t.

$$
a \equiv a_{1} \quad \bmod H_{k} \quad \text { and } \quad a \equiv a_{2} \quad \bmod H_{k} .
$$

Using a proper evaluation, one sees that $a$ is in $H_{k} \mathbb{A}\left(y_{k}\right)^{\times}$.

We present a detailed proof of the third assertion due to the presence of both $\mathbb{A}$ and $\mathbb{B}$, though the idea goes along the same line as before. It suffices to show that the intersection of $G_{j} \mathbb{A}^{\times} \mathbb{B}\left(Z_{1}\right)^{\times}$and $G_{j} \mathbb{A}^{\times} \mathbb{B}\left(Z_{2}\right)^{\times}$is a subset of $G_{j} \mathbb{A}^{\times} \mathbb{B}^{\times}$. Assume that $a$ is in the intersection. Then

$$
a \equiv a_{1} b_{1} \quad \bmod G_{j} \quad \text { and } \quad a \equiv a_{2} b_{2} \quad \bmod G_{j}
$$

for some $a_{1}, a_{2}$ in $\mathbb{A}^{\times}, b_{1}$ in $\mathbb{B}\left(Z_{1}\right)^{\times}$, and $b_{2}$ in $\mathbb{B}\left(Z_{2}\right)^{\times}$. Let $Z_{2}^{\prime}=Z_{2} \backslash \mathbb{B}$, and $c$ be a proper evaluation of $a_{1} /\left(a_{2} b_{2}\right)$ w.r.t. $Z_{2}^{\prime}$. Then $c b_{1}$ is a proper evaluation of $a_{1} b_{1} /\left(a_{2} b_{2}\right)$ w.r.t. $Z_{2}^{\prime}$, as $b_{1}$ is free of $Z_{2}^{\prime}$. So $c b_{1}$ is in $G_{j}$ by Remark 4.3 . Since $c$ is in $\mathbb{A}^{\times} \mathbb{B}^{\times}, b_{1}$ is in $G_{j} \mathbb{A}^{\times} \mathbb{B}^{\times}$, and so is $a$.

The next lemma says that some compatible rational functions belong to a common coset.

Lemma 4.3. Let $v_{1}, \ldots, v_{m}, w_{1}, \ldots, w_{n} \in \mathbb{F}(\mathbf{t}, \mathbf{x}, \mathbf{y})^{\times}$. Assume that the compatibility conditions in (4) and (5) hold.

(i) If $v_{j}$ is in $G_{j} \mathbb{F}(\mathbf{t}, \mathbf{y})^{\times} \mathbb{F}(\mathbf{x}, \mathbf{y})^{\times}$for all $j$ with $1 \leq j \leq m$, then there exists $f \in \mathbb{F}(\mathbf{t}, \mathbf{x}, \mathbf{y})$ s.t. each $v_{j}$ is in the coset $\ell \sigma_{j}(f) \mathbb{F}(\mathbf{t}, \mathbf{y})^{\times} \mathbb{F}(\mathbf{x}, \mathbf{y})^{\times}$.

(ii) Let $\mathbb{E}$ be a subfield of $\mathbb{F}(\mathbf{t}, \mathbf{x})$. If $w_{k} \in H_{k} \mathbb{E}(\mathbf{y})^{\times}$for all $k$ with $1 \leq k \leq n$, then there exists $f \in \mathbb{F}(\mathbf{t}, \mathbf{x}, \mathbf{y})$ s.t. each $w_{k}$ is in the coset $\ell \tau_{k}(f) \mathbb{E}(\mathbf{y})^{\times}$.

Proof. We are going to show the second assertion. The first one can be proved in the same fashion.

The second assertion clearly holds when $n=1$. Assume that $n>1$ and the lemma holds for $n-1$. Then there exist $g \in \mathbb{F}(\mathbf{t}, \mathbf{x}, \mathbf{y})$ and $b_{1}, \ldots, b_{n-1} \in \mathbb{E}(\mathbf{y})$ s.t. $w_{k}=\ell \tau_{k}(g) b_{k}$ for all $k$ with $1 \leq k \leq n-1$. Assume

$$
w_{n}=\ell \tau_{n}(g) a \text { for some } a \in \mathbb{F}(\mathbf{t}, \mathbf{x}, \mathbf{y}) .
$$

Then the compatibility conditions in (5) imply that the first-order system $\left\{\tau_{k}(z)=\ell \tau_{n}\left(b_{k}\right) z \mid k=1, \ldots, n-1\right\}$ has a solution $a$ in $\mathbb{F}(\mathbf{t}, \mathbf{x}, \mathbf{y})^{\times}$. It follows from the hypothesis $b_{k} \in \mathbb{E}(\mathbf{y})$ for all $k$ with $1 \leq k \leq n-1$ that the above system has a solution $a^{\prime}$ in $\mathbb{E}(\mathbf{y})^{\bar{x}}$. Thus, $a=c a^{\prime}$ for some constant $c$ w.r.t. $\tau_{1}, \ldots, \tau_{n-1}$. Consequently, $c$ belongs to $\mathbb{F}\left(\mathbf{t}, \mathbf{x}, y_{n}\right)$. On one hand, (17) leads to

$$
w_{n}=\ell \tau_{n}(g) c a^{\prime} \text {. }
$$

On the other hand, $w_{n} \in H_{n} \mathbb{E}(\mathbf{y})^{\times}$implies $c=\ell \tau_{n}(s) r$ for some $r$ in $\mathbb{E}(\mathbf{y})$ and $s$ in $\mathbb{F}(\mathbf{t}, \mathbf{x}, \mathbf{y})$. Let $Z^{\prime}=\left\{y_{1}, \ldots, y_{n-1}\right\}$, and let $s^{\prime}$ and $r^{\prime}$ be two proper evaluations of $s$ and $r$ w.r.t. $Z^{\prime}$ at a point in $\mathbb{F}^{n-1}$, respectively. Then $c=\ell \tau_{n}\left(s^{\prime}\right) r^{\prime}$ since $c$ is free of $Z^{\prime}$. By (18), $w_{n}=\ell \tau_{n}\left(s^{\prime} g\right) r^{\prime} a^{\prime}$. Set $f=s^{\prime} g$ and $b_{n}=r^{\prime} a^{\prime}$. Then $w_{k}=\ell \tau_{k}(f) b_{k}$ for all $k$ with $1 \leq k \leq n$, as $s^{\prime}$ is a constant w.r.t. $\tau_{1}, \ldots, \tau_{n-1}$.

\section{A STRUCTURE THEOREM}

In this section, we extend Lemmas 3.1, 3.2 and 3.3, and then combine these results to a structure theorem on $\Delta$ compatible rational functions.

The first proposition extends Lemma 3.1.

Proposition 5.1. Let $u_{1}, \ldots, u_{l}, v_{1}, \ldots, v_{m}$ be rational functions in $\mathbb{F}(\mathbf{t}, \mathbf{x}, \mathbf{y})$ with $v_{1} \cdots v_{m} \neq 0$. If the compatibility conditions in (3), (4) and (6) hold, then there exist $f$ in $\mathbb{F}(\mathbf{t}, \mathbf{x}, \mathbf{y}), a_{1}, \ldots, a_{m}, b_{1}, \ldots, b_{l}$ in $\mathbb{F}(\mathbf{t}, \mathbf{y})$, and $c_{1}, \ldots, c_{m}$ in $\mathbb{F}(\mathbf{x}, \mathbf{y})$ s.t., for all $i$ with $1 \leq i \leq l$,

$$
u_{i}=\ell \delta_{i}(f)+\ell \delta_{i}\left(a_{1}\right) x_{1}+\cdots+\ell \delta_{i}\left(a_{m}\right) x_{m}+b_{i},
$$

and, for all $j$ with $1 \leq j \leq m$,

$$
v_{j}=\ell \sigma_{j}(f) a_{j} c_{j} .
$$

Moreover, the sequence $b_{1}, \ldots, b_{l}, c_{1}, \ldots, c_{m}$ is compatible w.r.t. $\left\{\delta_{1}, \ldots, \delta_{l}, \sigma_{1}, \ldots, \sigma_{m}\right\}$.

Proof. First, we consider the case in which $l=1$ and $m$ arbitrary. The proposition holds when $m=1$ by Lemma 3.1 . Assume that $m>1$ and the proposition holds for the values lower than $m$. Applying the induction hypothesis to $t_{1}, x_{1}$, $\ldots, x_{m-1}$ and to $t_{1}, x_{2}, \ldots, x_{m}$, respectively, we see that both $u_{1} \in N_{1, m}$ and $u_{1} \in N_{1,1}$. Since $m>1, u_{1} \in N_{1}$ by Lemma 4.1 (ii). Hence,

$$
u_{1}=\ell \delta_{1}(f)+\ell \delta_{1}\left(a_{1}\right) x_{1}+\cdots+\ell \delta_{1}\left(a_{m}\right) x_{m}+b_{1}
$$

for some $f \in \mathbb{F}\left(t_{1}, \mathbf{x}, \mathbf{y}\right)$ and $a_{1}, \ldots, a_{m}, b_{1} \in \mathbb{F}\left(t_{1}, \mathbf{y}\right)$. Assume that $v_{j}=\ell \sigma_{j}(f) a_{j} c_{j}$. Then $c_{1}, \ldots, c_{m}$ are in $\mathbb{F}(\mathbf{x}, \mathbf{y})$ by the compatibility conditions in (6) (see Example 3.2). The proposition holds for $l=1$ and $m$ arbitrary.

Second, we show that the proposition holds for all $l$ and $m$ by induction on $l$. It holds if $l=1$ by the preceding paragraph. Assume that $l>1$ and that the proposition holds for the values lower than $l$. Applying the induction hypothesis to $t_{1}, \ldots, t_{l-1}, \mathbf{x}$ and to $t_{2}, \ldots, t_{l}, \mathbf{x}$, respectively, we have

$$
v_{j} \in\left(G_{j} \mathbb{A}^{\times} \mathbb{B}\left(Z_{1}\right)^{\times}\right) \cap\left(G_{j} \mathbb{A}^{\times} \mathbb{B}\left(Z_{2}\right)^{\times}\right),
$$

where $\mathbb{A}=\mathbb{F}(\mathbf{t}, \mathbf{y}), \mathbb{B}=\mathbb{F}(\mathbf{x}, \mathbf{y}), Z_{1}=\left\{t_{l}\right\}$, and $Z_{2}=\left\{t_{1}\right\}$. We see that $v_{j} \in G_{j} \mathbb{A}^{\times} \mathbb{B}^{\times}$by Lemma 4.2 (iii). So $v_{j} \in \ell \sigma_{j}(f) \mathbb{A}^{\times} \mathbb{B}^{\times}$ for some $f$ in $\mathbb{F}(\mathbf{t}, \mathbf{x}, \mathbf{y})$ by Lemma 4.3 (i). Thus,

$$
v_{j}=\ell \sigma_{j}(f) a_{j} c_{j},
$$

where $a_{j} \in \mathbb{A}, c_{j} \in \mathbb{B}$ and $j=1, \ldots, m$. Assume that, for all $i$ with $1 \leq i \leq l, u_{i}=\ell \delta_{i}(f)+\sum_{j=1}^{m} \ell \delta_{i}\left(a_{j}\right) x_{j}+b_{i}$. All the $b_{i}$ 's belong to $\mathbb{F}(\mathbf{t}, \mathbf{y})$ by the compatibility conditions in (6) (see Example 3.2). The sequence $b_{1}, \ldots, b_{l}, c_{1}, \ldots, c_{m}$ is compatible because of (3), (4) and (6).

The second proposition extends Lemma 3.2.

Proposition 5.2. Let $u_{1}, \ldots, u_{l}, w_{1}, \ldots, w_{n}$ be rational functions in $\mathbb{F}(\mathbf{t}, \mathbf{x}, \mathbf{y})$ with $w_{1} \cdots w_{n} \neq 0$. Assume that the compatibility conditions (3), (5) and (7) hold. Then 
there exist $f$ in $\mathbb{F}(\mathbf{t}, \mathbf{x}, \mathbf{y}), a_{1}, \ldots, a_{l}$ in $\mathbb{F}(\mathbf{t}, \mathbf{x})$, and $b_{1}, \ldots, b_{n}$ in $\mathbb{F}(\mathbf{x}, \mathbf{y})$ s.t.

$$
u_{i}=\ell \delta_{i}(f)+a_{i} \quad \text { and } \quad w_{k}=\ell \tau_{k}(f) b_{k}
$$

for all $i$ with $1 \leq i \leq l$ and $k$ with $1 \leq k \leq n$. Moreover, the sequence $a_{1}, \ldots, a_{l}, b_{1}, \ldots, b_{n}$ is compatible w.r.t. the set $\left\{\delta_{1}, \ldots, \delta_{l}, \tau_{1}, \ldots, \tau_{n}\right\}$.

The proof of this proposition goes along the same line as in that of Proposition 5.1.

The last proposition extends Lemma 3.3.

Proposition 5.3. Let $v_{1}, \ldots, v_{m}, w_{1}, \ldots, w_{n}$ be rational functions in $\mathbb{F}(\mathbf{t}, \mathbf{x}, \mathbf{y})^{\times}$. Assume that the compatibility conditions in (4), (5) and (8) hold. Then there exist a rational function $f$ in $\mathbb{F}(\mathbf{t}, \mathbf{x}, \mathbf{y}), a_{1}, \ldots, a_{m}$ in $\mathbb{F}(\mathbf{t}, \mathbf{x})$, and $b_{1}, \ldots, b_{n}$ in $\mathbb{F}(\mathbf{t}, \mathbf{y})$ s.t., for all $j$ with $1 \leq j \leq m$ and $k$ with $1 \leq k \leq n$,

$$
v_{j}=\ell \sigma_{j}(f) a_{j} \quad \text { and } \quad w_{k}=\ell \tau_{k}(f) b_{k} .
$$

Furthermore, the sequence $a_{1}, \ldots, a_{m}, b_{1}, \ldots, b_{n}$ is compatible w.r.t. $\left\{\sigma_{1}, \ldots, \sigma_{m}, \tau_{1}, \ldots, \tau_{n}\right\}$.

Proof. First, we consider the case, in which $m=1$ and $n$ arbitrary. We proceed by induction on $n$. The proposition holds when $n=1$ by Lemma 3.3. Assume that $n>1$, and the proposition holds for the values lower than $n$. Applying the induction hypothesis to $x_{1}, y_{1}, \ldots, y_{n-1}$ and to $x_{1}, y_{2}, \ldots, y_{n}$, respectively, we get $v_{1} \in G_{1} \mathbb{F}\left(\mathbf{t}, x_{1}, y_{n}\right)^{\times} \cap G_{1} \mathbb{F}\left(\mathbf{t}, x_{1}, y_{1}\right)^{\times}$. Setting $\mathbb{A}=\mathbb{F}(\mathbf{t}), Z_{1}=\left\{y_{n}\right\}$ and $Z_{2}=\left\{y_{1}\right\}$ in Lemma 4.2 (i), we see that $v_{1} \in G_{1} \mathbb{F}\left(\mathbf{t}, x_{1}\right)^{\times}$, which, together with the definition of $G_{1} \mathbb{F}\left(\mathbf{t}, x_{1}\right)^{\times}$, there exist $f$ in $\mathbb{F}\left(\mathbf{t}, x_{1}, \mathbf{y}\right)$ and $a$ in $\mathbb{F}\left(\mathbf{t}, x_{1}\right)$ s.t. $v_{1}=\ell \sigma_{1}(f) a$. Assume that $w_{k}=\ell \tau_{k}(f) b_{k}$ for some $b_{k}$ in $\mathbb{F}\left(\mathbf{t}, x_{1}, \mathbf{y}\right)$ and for all $k$ with $1 \leq k \leq n$. By (8), $\sigma_{1}\left(b_{k}\right)=b_{k}$, i.e., $b_{k} \in \mathbb{F}(\mathbf{t}, \mathbf{y})$ (see Example 3.4). The proposition holds for $m=1$ and $n$ arbitrary.

Second, assume that $m>1$ and the proposition holds for values lower than $m$ and arbitrary $n$. Applying this induction hypothesis to $x_{1}, \ldots, x_{m-1}, \mathbf{y}$ and to $x_{2}, \ldots, x_{m}, \mathbf{y}$, respectively, we have

$$
w_{k} \in\left(H_{k} \mathbb{A}\left(y_{k}, Z_{1}\right)^{\times}\right) \cap\left(H_{k} \mathbb{A}\left(y_{k}, Z_{2}\right)^{\times}\right),
$$

where $\mathbb{A}=\mathbb{F}\left(\mathbf{t}, y_{1}, \ldots, y_{k-1}, y_{k+1}, \ldots, y_{n}\right), Z_{1}$ and $Z_{2}$ are equal to $\left\{x_{m}\right\}$ and $\left\{x_{1}\right\}$, respectively. Thus, $w_{k} \in H_{k} \mathbb{A}\left(y_{k}\right)^{\times}$ by Lemma 4.2 (ii), and $w_{k} \in \ell \tau_{k}(f) \mathbb{A}\left(y_{k}\right)^{\times}$for some $f$ in $\mathbb{F}(\mathbf{t}, \mathbf{x}, \mathbf{y})$ by Lemma 4.3 (ii). Let $w_{k}=\ell \tau_{k}(f) b_{k}$, where $b_{k}$ is in $\mathbb{A}\left(y_{k}\right)^{\times}$, and $k=1, \ldots, n$. Let $a_{j}=v_{j} / \ell \sigma_{j}(f)$ for all $j$ with $1 \leq j \leq m$. Then $\tau_{k}\left(a_{j}\right)=a_{j}$ for all $k$ with $1 \leq k \leq n$ and $j$ with $1 \leq j \leq m$ by the compatibility conditions in (8) (see Example 3.4). Hence, all the $a_{j}$ 's are in $\mathbb{F}(\mathbf{t}, \mathbf{x})$. The sequence $a_{1}, \ldots, a_{m}, b_{1}, \ldots, b_{n}$ is compatible because of (4), (5) and (8).

Now, we present a theorem describing the structure of compatible rational functions.

TheOREM 5.4. Let

$$
u_{1}, \ldots, u_{l}, v_{1}, \ldots, v_{m}, w_{1}, \ldots, w_{n}
$$

be a sequence of rational functions in $\mathbb{F}(\mathbf{t}, \mathbf{x}, \mathbf{y})$. If the sequence is $\Delta$-compatible, then there exist $f$ in $\mathbb{F}(\mathbf{t}, \mathbf{x}, \mathbf{y}), \alpha_{1}$, $\ldots, \alpha_{m}, \beta_{1}, \ldots, \beta_{l}$ in $\mathbb{F}(\mathbf{t}), \lambda_{1}, \ldots, \lambda_{m}$ in $\mathbb{F}(\mathbf{x})$, and $\mu_{1}$, $\ldots, \mu_{n}$ in $\mathbb{F}(\mathbf{y})$ s.t., for all $i$ with $1 \leq i \leq l$,

$$
u_{i}=\ell \delta_{i}(f)+\ell \delta_{i}\left(\alpha_{1}\right) x_{1}+\cdots+\ell \delta_{i}\left(\alpha_{m}\right) x_{m}+\beta_{i},
$$

for all $j$ with $1 \leq j \leq m$, and, for all $k$ with $1 \leq k \leq n$,

$$
v_{j}=\ell \sigma_{j}(f) \alpha_{j} \lambda_{j} \quad \text { and } \quad w_{k}=\ell \tau_{k}(f) \mu_{k} .
$$

Moreover, the sequence $\beta_{1}, \ldots, \beta_{l}, \lambda_{1}, \ldots, \lambda_{m}, \mu_{1}, \ldots, \mu_{n}$ is $\Delta$-compatible.

Proof. By Propositions 5.2 and 5.3,

$$
w_{k}=\ell \tau_{k}\left(g^{\prime}\right) a_{k}^{\prime}=\ell \tau_{k}(\tilde{g}) \tilde{a}_{k}
$$

for some $g^{\prime}, \tilde{g} \in \mathbb{F}(\mathbf{t}, \mathbf{x}, \mathbf{y}), a_{k}^{\prime} \in \mathbb{F}(\mathbf{x}, \mathbf{y})$, and $\tilde{a}_{k} \in \mathbb{F}(\mathbf{t}, \mathbf{y})$ with $1 \leq k \leq n$. Set $Z_{1}=\left\{t_{1}, \ldots, t_{l}\right\}, Z_{2}=\left\{x_{1}, \ldots, x_{m}\right\}$, and $\mathbb{A}=\mathbb{F}\left(y_{1}, \ldots, y_{k-1}, y_{k+1}, \ldots, y_{n}\right)$ in Lemma 4.2 (ii). Then the lemma implies that there exist $\mu_{k}$ in $\mathbb{F}(\mathbf{y})$ and $g_{k}$ in $\mathbb{F}(\mathbf{t}, \mathbf{x}, \mathbf{y})$ s.t. $w_{k}=\ell \tau_{k}\left(g_{k}\right) \mu_{k}$. Setting $\mathbb{E}=\mathbb{F}$ in the second assertion of Lemma 4.3, we may further assume that all the $g_{k}$ 's are equal to a rational function, say $g$. Let

$u_{i}=\ell \delta_{i}(g)+r_{i}(1 \leq i \leq l)$ and $v_{j}=\ell \sigma_{j}(g) s_{j}(1 \leq j \leq m)$.

Then the compatibility conditions in (7) imply that the $r_{i}$ 's are in $\mathbb{F}(\mathbf{t}, \mathbf{x})$ (see Example 3.3). Similarly, those conditions in (8) imply that the $s_{j}$ 's are in $\mathbb{F}(\mathbf{t}, \mathbf{x})$ (see Example 3.4). Furthermore, $r_{1}, \ldots, r_{l}, s_{1}, \ldots, s_{m}$ are compatible w.r.t. the set $\left\{\delta_{1}, \ldots, \delta_{l}, \sigma_{1}, \ldots, \sigma_{m}\right\}$. By Proposition 5.1, we get

$$
r_{i}=\ell \delta_{i}(b)+\ell \delta_{i}\left(\alpha_{1}\right) x_{1}+\cdots+\ell \delta_{i}\left(\alpha_{m}\right) x_{m}+\beta_{i},
$$

and $s_{j}=\ell \sigma_{j}(b) \alpha_{j} \lambda_{j}$ for some $b$ in $\mathbb{F}(\mathbf{t}, \mathbf{x}), \alpha_{j}, \beta_{i}$ in $\mathbb{F}(\mathbf{t}), \lambda_{j}$ in $\mathbb{F}(\mathbf{x}), 1 \leq i \leq l$, and $1 \leq j \leq m$. Note that $b$ belongs to $\mathbb{F}(\mathbf{t}, \mathbf{x})$. Setting $f=g b$, we get the desired form for $u_{i}$ 's, $v_{j}$ 's and $w_{k}$ 's. The compatibility of the sequence $\beta_{1}, \ldots, \beta_{l}$, $\lambda_{1}, \ldots, \lambda_{m}, \mu_{1}, \ldots, \mu_{n}$ follows from that of $u_{1}, \ldots, u_{l}, v_{1}$, $\ldots, v_{m}, w_{1}, \ldots, w_{n}$.

With the notation introduced in Theorem 5.4, we say that the sequence:

$$
f, \alpha_{1}, \ldots, \alpha_{m}, \beta_{1}, \ldots, \beta_{l}, \lambda_{1}, \ldots, \lambda_{m}, \mu_{1}, \ldots, \mu_{n}
$$

is a representation of $\Delta$-compatible rational functions given in (19) if the equalities in (20) and (21) hold.

A rational function $\mathbb{F}(\mathbf{t}, \mathbf{x}, \mathbf{y})$ is said to be nonsplit w.r.t. $\mathbf{t}$ if its denominator and numerator have no irreducible factors in $\mathbb{F}[\mathbf{t}]$. Similarly, we define the notion of nonsplitness w.r.t. $\mathbf{x}$ or $\mathbf{y}$. Let $\prec$ be a fixed monomial ordering on $\mathbb{F}[\mathbf{t}, \mathbf{x}, \mathbf{y}]$. A nonzero rational function in $\mathbb{F}(\mathbf{t}, \mathbf{x}, \mathbf{y})$ is said to be monic w.r.t. $\prec$ if its denominator and numerator are both monic w.r.t. $\prec$. A representation (22) of $\Delta$-compatible rational functions in (19) is said to be standard w.r.t. $\prec$ if

(i) $f$ is nonsplit w.r.t. $\mathbf{t}, \mathbf{x}$, and $\mathbf{y}$, that is, the nontrivial irreducible factors of $\operatorname{den}(f) \operatorname{num}(f)$ are neither in $\mathbb{F}[\mathbf{t}]$, nor in $\mathbb{F}[\mathbf{x}]$, nor in $\mathbb{F}[\mathbf{y}]$;

(ii) both $f$ and $\alpha_{j}$ are monic w.r.t. $\prec, j=1,2, \ldots, m$.

Assume that the sequence (22) is a representation of (19). Factor $f=f_{1} f_{2} f_{3} f_{4}$, where $f_{1}$ is monic and nonsplit w.r.t. $\mathbf{t}, \mathbf{x}$ and $\mathbf{y}, f_{2}$ is in $\mathbb{F}(\mathbf{t}), f_{3}$ in $\mathbb{F}(\mathbf{x})$, and $f_{4}$ in $\mathbb{F}(\mathbf{y})$. Set $\alpha_{j}=c_{j} \alpha_{j}^{\prime}$, where $c_{j} \in \mathbb{F}$, and $\alpha_{j}^{\prime}$ is monic. Then

$$
f_{1}, \alpha_{1}^{\prime}, \ldots, \alpha_{m}^{\prime}, \beta_{1}+\ell \delta_{1}\left(f_{2}\right), \ldots, \beta_{l}+\ell \delta_{l}\left(f_{2}\right),
$$

$\ell \sigma_{1}\left(f_{3}\right) c_{1} \lambda_{1}, \ldots, \ell \sigma_{m}\left(f_{3}\right) c_{m} \lambda_{m}, \ell \tau_{1}\left(f_{4}\right) \mu_{1}, \ldots, \ell \tau_{n}\left(f_{4}\right) \mu_{n}$ is also a representation of (19). This proves the existence of standard representations. Its uniqueness follows from the uniqueness of factorization of rational functions.

Corollary 5.5. A $\Delta$-compatible sequence has a unique standard representation w.r.t. a given monomial ordering. 


\section{ALGORITHMS AND APPLICATIONS}

In this section, we discuss how to compute a representation of compatible rational functions, and present two applications in analyzing $H$-solutions. Let us fix a monomial ordering on $\mathbb{F}[\mathbf{t}, \mathbf{x}, \mathbf{y}]$ for standard representations.

Let the sequence given in (19) be $\Delta$-compatible. We compute a representation of the sequence in the form of (22).

First, we compute $\mu_{1}(\mathbf{y}), \ldots, \mu_{n}(\mathbf{y})$ in the sequence (22). By gcd-computation, we write $w_{k}=a_{k} b_{k}$, where $a_{k}$ is nonsplit w.r.t. $\mathbf{y}, b_{k}$ is in $\mathbb{F}(\mathbf{y})$, and $k=1, \ldots, n$. By Theorem 5.4, $w_{k}=\ell \tau_{k}(f) \mu_{k}$, where $f$ is nonsplit w.r.t. $\mathbf{y}$ and $\mu_{k}$ is in $\mathbb{F}(\mathbf{y})$. Thus, $b_{k}=c_{k} \mu_{k}$ for some $c_{k} \in \mathbb{F}^{\times}$.

To determine $c_{k}$, write $a_{k}=\ell \tau_{k}\left(g_{k}\right) r_{k}$, where $g_{k}$ and $r_{k}$ are in $\mathbb{F}(\mathbf{t}, \mathbf{x}, \mathbf{y})$ with $r_{k}$ being $\tau_{k}$-reduced. By the two expressions of $w_{k}, c_{k} r_{k}=\ell \tau_{k}\left(f / g_{k}\right)$. Since $a_{k}$ is nonsplit w.r.t. $\mathbf{y}$ and $r_{k}$ is $\tau_{k}$-reduced, $g_{k}$ can be chosen to be nonsplit w.r.t. $\mathbf{y}$, and so is $f / g_{k}$. Thus, $f / g_{k}$ is free of $y_{k}$, because $c_{k} r_{k}$ is $\tau_{k}$-reduced. Accordingly, $c_{k} r_{k}=1$ and $\mu_{k}=r_{k} b_{k}$. As a byproduct, we obtain $g_{k}$ with $\ell \tau_{k}(f)=\ell \tau_{k}\left(g_{k}\right)$.

Second, we compute $\alpha_{1}, \ldots, \alpha_{m}$ and $\lambda_{1}, \ldots, \lambda_{m}$. Assume that $j$ is an integer with $1 \leq j \leq m$. By gcd-computation, we write $v_{j}=s_{j} a_{j} b_{j}$, where $s_{j}$ is nonsplit w.r.t. $\mathbf{t}$ and $\mathbf{x}, a_{j}$ is in $\mathbb{F}(\mathbf{t})$, and $b_{j}$ in $\mathbb{F}(\mathbf{x})$. Moreover, set $a_{j}$ to be monic. By Theorem 5.4, $v_{j}=\ell \sigma_{j}(f) \alpha_{j} \lambda_{j}$, where $f$ is nonsplit w.r.t. $\mathbf{t}$ and $\mathbf{x}, \alpha_{j}$ is a monic element in $\mathbb{F}(\mathbf{t})$, and $\lambda_{j}$ is in $\mathbb{F}(\mathbf{x})$. Hence, $a_{j}=\alpha_{j}$ and $b_{j}=c_{j} \lambda_{j}$ for some $c_{j} \in \mathbb{F}^{\times}$. As in the preceding paragraph, we write $s_{j}=\ell \sigma_{j}\left(g_{j}^{\prime}\right) r_{j}$ with $r_{j}$ being $\sigma_{j}$-reduced. Then $c_{j} r_{j}=\ell \sigma_{j}\left(f / g_{j}^{\prime}\right)$. Since $c_{j} r_{j}$ is $\sigma_{j}$ reduced, $c_{j} r_{j}=1$. Hence, $\lambda_{j}=r_{j} b_{j}$. As a byproduct, we find $g_{j}^{\prime}$ with $\ell \sigma_{j}(f)=\ell \sigma_{j}\left(g_{j}^{\prime}\right)$

Third, we compute $f$. Note that $f$ is a nonzero rational solution of the system $\left\{\sigma_{j}(z)=\ell \sigma_{j}\left(g_{j}^{\prime}\right) z, \tau_{k}(z)=\ell \tau_{k}\left(g_{k}\right) z\right\}$, where $1 \leq j \leq m, 1 \leq k \leq n$, and $g_{j}^{\prime}, g_{k}$ are obtained in the first two steps. So $f$ can be computed by several methods, e.g., the method in the proof of [14, Proposition 3].

At last, we set $\beta_{i}=u_{i}-\ell \delta_{i}(f)-\sum_{j=1}^{m} \ell \delta_{i}\left(\alpha_{j}\right) x_{j}$, for all $i$ with $1 \leq i \leq l$. Using $v_{j}=\ell \sigma_{j}(f) \alpha_{j} \lambda_{j}$ and $w_{k}=\ell \tau_{k}(f) \mu_{k}$ and the compatibility conditions in (6) and (7), we see that all the $\beta_{i}$ 's are in $\mathbb{F}(\mathbf{t})$, as required.

Example 6.1. Consider the case $l=m=n=1$. Let $u$, $v$ and $w$ be compatible rational functions, where

$$
\begin{aligned}
& u=\frac{\left(4 t+2 x+y^{2}\right)(t+1)+(t+x+1)(t+x)\left(2 t+y^{2}\right)}{(t+1)(t+x)\left(2 t+y^{2}\right)}, \\
& v=\frac{2(2 x+3)(x+1)(t+1)(t+x+1)(5 x+y)}{(5 x+y+5)(t+x)}, \\
& w=\frac{(5 x+y)\left(2 t+q^{2} y^{2}\right)(1+q y)}{(5 x+q y)\left(2 t+y^{2}\right)} .
\end{aligned}
$$

$A$ representation of $u, v, w$ is of the form

$$
\left(\frac{\left(2 t+y^{2}\right)(t+x)}{5 x+y}, t+1,1,2(2 x+3)(x+1), q y+1\right) .
$$

From now on, we assume that our ground field $\mathbb{F}$ is algebraically closed. In general, $\Delta$-extensions of $\mathbb{F}(\mathbf{t}, \mathbf{x}, \mathbf{y})$ are rings. We recall that an $H$-solution over $\mathbb{F}(\mathbf{t}, \mathbf{x}, \mathbf{y})$ is a nonzero solution of system (1) and, given a finite number of $H$-solutions, there is a $\Delta$-extension of $\mathbb{F}(\mathbf{t}, \mathbf{x}, \mathbf{y})$ containing these $H$-solutions and their inverses. The ring of constants of this $\Delta$-extension is equal to $\mathbb{F}$ by Theorem 2 in [5]. We will only encounter finitely many pairwise dissimilar $H$-solutions. Hence, it makes sense to multiply and invert them in some $\Delta$-extension, which will not be specified explicitly if no ambiguity arises. All $H$-solutions we consider will be over $\mathbb{F}(\mathbf{t}, \mathbf{x}, \mathbf{y})$. Denote by $\mathbf{0}_{s}$ and $\mathbf{1}_{s}$ the sequences consisting of $s 0$ 's and of $s$ 1's, respectively.

An $H$-solution is said to be a symbolic power if its certificates are of the form

$$
\sum_{j=1}^{m} x_{j} \ell \delta_{1}\left(\alpha_{j}\right), \ldots, \sum_{j=1}^{m} x_{j} \ell \delta_{l}\left(\alpha_{j}\right), \alpha_{1}, \ldots, \alpha_{m}, \mathbf{1}_{n},
$$

where $\alpha_{1}, \ldots, \alpha_{m}$ are monic elements in $\mathbb{F}(\mathbf{t})^{\times}$. It is easy to verify that such a sequence is $\Delta$-compatible. Such a symbolic power is denoted $\alpha_{1}^{x_{1}} \cdots \alpha_{m}^{x_{m}}$. The monicity of the $\alpha_{i}$ 's excludes the case, in which some $\alpha_{i}$ is a constant different from one. By an $E$-solution, we mean an $H$-solution whose certificates are of the form $\beta_{1}, \ldots, \beta_{l}, \mathbf{1}_{m+n}$, where $\beta_{1}, \ldots, \beta_{l}$ are in $\mathbb{F}(\mathbf{t})$. An $E$-solution is a hyperexponential function w.r.t. the derivations, and a constant w.r.t. other operators. By a $G$-solution, we mean an $H$-solution whose certificates are of the form $\mathbf{0}_{l}, \lambda_{1}, \ldots, \lambda_{m}, \mathbf{1}_{n}$, where $\lambda_{1}, \ldots, \lambda_{m}$ are in $\mathbb{F}(\mathbf{x})^{\times}$. A $G$-solution is a hypergeometric term w.r.t. the shift operators, and a constant w.r.t. other operators. Similarly, by a $Q$-solution, we mean an $H$-solution whose certificates are of the form $\mathbf{0}_{l}, \mathbf{1}_{m}, \mu_{1}, \ldots, \mu_{n}$, where $\mu_{1}, \ldots, \mu_{n}$ are in $\mathbb{F}(\mathbf{y})^{\times}$. A $Q$-solution is a $q$-hypergeometric term w.r.t. the $q$-shift operators, and a constant w.r.t. other operators.

The next proposition describes a multiplicative decomposition of $H$-solutions.

Proposition 6.1. An $\mathrm{H}$-solution is a product of an element in $\mathbb{F}^{\times}$, a rational function in $\mathbb{F}(\mathbf{t}, \mathbf{x}, \mathbf{y})$, a symbolic power, an E-solution, a $G$-solution, and a $Q$-solution.

Proof. Let $h$ be an $H$-solution. Then its certificates are compatible. By Theorem 5.4, the certificates have a standard representation $f, \alpha_{1}, \ldots, \alpha_{m}, \beta_{1}, \ldots, \beta_{l}, \lambda_{1}, \ldots, \lambda_{m}$, $\mu_{1}, \ldots, \mu_{n}$. Moreover, the following three sequences:

$$
\beta_{1}, \ldots, \beta_{l}, \mathbf{1}_{m+n} ; \quad \mathbf{0}_{l}, \lambda_{1}, \ldots, \lambda_{m}, \mathbf{1}_{n} ; \quad \mathbf{0}_{l}, \mathbf{1}_{m}, \mu_{1}, \ldots, \mu_{n}
$$

are $\Delta$-compatible, respectively. Hence, there exist an $E$ solution $\mathcal{E}$, a $G$-solution $\mathcal{G}$, and a $Q$-solution $\mathcal{Q}$ s.t. their certificates are given in the above three sequences, respectively. It follows from Theorem 5.4 that $h$ and the product $f \alpha_{1}^{x_{1}} \cdots \alpha_{m}^{x_{m}} \mathcal{E} \mathcal{G} \mathcal{Q}$ have the same certificates. So they differ by a multiplicative constant, which is in $\mathbb{F}$.

The $H$-solution in Example 6.1 can be decomposed as

$$
\frac{\left(2 t+y^{2}\right)(t+x)}{5 x+y}(t+1)^{x} \exp (t)(2 x+1) ! \Gamma_{q}(1+q y),
$$

where $\Gamma_{q}(1+q y)$ is a $Q$-solution with certificates $0,1,1+q y$.

The next proposition characterizes rational $H$-solutions via their standard representations.

Proposition 6.2. Let $\mathcal{P}$ be a symbolic power, $\mathcal{E}$ an $E$ solution, $\mathcal{G}$ a $G$-solution and $\mathcal{Q}$ a $Q$-solution. Then $\mathcal{P} \mathcal{E} \mathcal{Q}$ is in $\mathbb{F}(\mathbf{t}, \mathbf{x}, \mathbf{y})$ iff $\mathcal{P} \in \mathbb{F}, \mathcal{E} \in \mathbb{F}(\mathbf{t}), \mathcal{G} \in \mathbb{F}(\mathbf{x})$ and $\mathcal{Q} \in \mathbb{F}(\mathbf{y})$.

Proof. $(\Leftarrow)$ Clear.

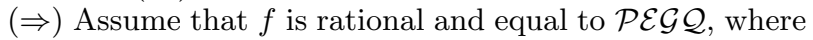
$\mathcal{P}, \mathcal{E}, \mathcal{G}, \mathcal{Q}$ are a symbolic power, an $E$-, a $G$-, and a $Q$ solution, respectively. Suppose that the certificates of $\mathcal{P}$ are given in (23). Applying $\ell \delta_{i}$ to $f, i=1, \ldots, l$, we see that

$$
\ell \delta_{i}(f)=\sum_{j=1}^{m} \ell \delta_{i}\left(\alpha_{j}\right) x_{j}+\ell \delta_{i}(\mathcal{E}) .
$$


Comparing the polynomial parts of the left and right handsides of the above equality w.r.t. $x_{j}$, we see that $\ell \delta_{i}\left(\alpha_{j}\right)=0$ by Remark 2.2 and $\ell \delta_{i}(\mathcal{E}) \in \mathbb{F}(\mathbf{t})$ for all $i$ and $j$. Hence, all the $\alpha_{j}$ 's are in $\mathbb{F}$, and, consequently, all the $\alpha_{j}$ 's are equal to one as they are monic. Hence, $\mathcal{P}$ is in $\mathbb{F}$. Moreover,

$$
\ell \delta_{i}(f)=\ell \delta_{i}(\mathcal{E}) \quad \text { for all } i \text { with } 1 \leq i \leq l .
$$

Let $g$ be a proper evaluation of $f$ w.r.t. $\mathbf{x}$ and $\mathbf{y}$. Then

$$
\ell \delta_{i}(g)=\ell \delta_{i}(\mathcal{E}) \quad \text { for all } i \text { with } 1 \leq i \leq l,
$$

since $\ell \delta_{i}(\mathcal{E})$ is in $\mathbb{F}(\mathbf{t})$. Hence, $\ell \delta_{i}(\mathcal{E} / g)=0, \ell \sigma_{j}(\mathcal{E} / g)=1$, and $\ell \tau_{k}(\mathcal{E} / g)=1$, where $1 \leq i \leq l, 1 \leq j \leq m$, and $1 \leq k \leq n$. We conclude that $\mathcal{E}=c g$ for some $c \in \mathbb{F}$. So $\mathcal{E}$ is in $\mathbb{F}(\mathbf{t})$.

Applying $\ell \sigma_{j}$ and $\ell \tau_{k}$ to $f$ leads to $\ell \sigma_{j}(f)=\ell \sigma_{j}(\mathcal{G})$, and $\ell \tau_{k}(f)=\ell \tau_{k}(\mathcal{Q})$, respectively. One can show that $\mathcal{G}$ is in $\mathbb{F}(\mathbf{x})$ and $\mathcal{Q}$ is in $\mathbb{F}(\mathbf{y})$ by similar arguments.

Now, we consider how to determine whether a finite number of $H$-solutions are algebraically dependent over $\mathbb{F}(\mathbf{t}, \mathbf{x}, \mathbf{y})$. Let $h_{1}, \cdots, h_{s}$ be $H$-solutions. By Proposition 6.1,

$$
h_{i} \equiv \mathcal{P}_{i} \mathcal{E}_{i} \mathcal{G}_{i} \mathcal{Q}_{i} \quad \bmod \mathbb{F}(\mathbf{t}, \mathbf{x}, \mathbf{y})^{\times}, \quad i=1, \ldots, s,
$$

where $\mathcal{P}_{i}, \mathcal{E}_{i}, \mathcal{G}_{i}, \mathcal{Q}_{i}$ are a symbolic power, an $E$-solution, a $G$-solution, and a $Q$-solution, respectively.

Corollary 6.3. Let $h_{1}, \ldots, h_{s}$ be $H$-solutions s.t. all the congruences in (24) hold. Then they are algebraically dependent over $\mathbb{F}(\mathbf{t}, \mathbf{x}, \mathbf{y})$ iff there exist integers $\omega_{1}, \ldots \omega_{s}$, not all zero, s.t. $\mathcal{P}_{1}^{\omega_{1}} \ldots \mathcal{P}_{s}^{\omega_{s}}$ is in $\mathbb{F}, \mathcal{E}_{1}^{\omega_{1}} \cdots \mathcal{E}_{s}^{\omega_{s}}$ in $\mathbb{F}(\mathbf{t}), \mathcal{G}_{1}^{\omega_{1}} \cdots \mathcal{G}_{s}^{\omega_{s}}$ in $\mathbb{F}(\mathbf{x})$ and $\mathcal{Q}_{1}^{\omega_{1}} \cdots \mathcal{Q}_{s}^{\omega_{s}}$ in $\mathbb{F}(\mathbf{y})$.

Proof. It follows from [15, Corollary 4.2] that $h_{1}, \cdots, h_{s}$ are algebraically dependent over $\mathbb{F}(\mathbf{t}, \mathbf{x}, \mathbf{y})$ iff there exist integers $\omega_{1}, \ldots \omega_{s}$, not all zero, s.t. $h_{1}^{\omega_{1}} \ldots h_{s}^{\omega_{s}}$ is in $\mathbb{F}(\mathbf{t}, \mathbf{x}, \mathbf{y})$. The corollary follows from (24) and Proposition 6.2.

By the above corollary, one may determine the algebraic dependence of $h_{1}, \ldots, h_{s}$ using the decompositions in Proposition 6.1. By gcd-computation, one can find all nonzero integer vectors $\left(\omega_{1}, \ldots \omega_{s}\right)$ s.t. $\mathcal{P}_{1}^{\omega_{1}} \ldots \mathcal{P}_{s}^{\omega_{s}}$ is in $\mathbb{F}$. According to [19], one can find all nonzero integer vectors $\left(\omega_{1}, \ldots \omega_{s}\right)$ s.t. $\mathcal{E}_{1}^{\omega_{1}} \ldots \mathcal{E}_{s}^{\omega_{s}} \in \mathbb{F}(\mathbf{t})$ by seeking rational number solutions of a linear homogeneous system over $\mathbb{F}$. Computing all nonzero integer vectors $\left(\omega_{1}, \ldots \omega_{s}\right)$ s.t. $\mathcal{G}_{1}^{\omega_{1}} \ldots \mathcal{G}_{s}^{\omega_{s}} \in \mathbb{F}(\mathbf{x})$ reduces to the following subproblem: given $c_{1}, \ldots, c_{s} \in \mathbb{F}^{\times}$, compute integers $\omega_{1}, \ldots \omega_{s}$, not all zero, with $c_{1}^{\omega_{1}} \cdots c_{s}^{\omega_{s}}=1$ (see [19]). Algorithms for tackling this subproblem and related discussions are contained in $[13, \S 7.3]$ and the references given there. We are trying to develop an algorithm that finds integers $\omega_{1}, \ldots \omega_{s}$, not all zero, s.t. $\mathcal{Q}_{1}^{\omega_{1}} \ldots \mathcal{Q}_{s}^{\omega_{s}}$ belongs to $\mathbb{F}(\mathbf{y})$.

The reader is referred to [6] for an extended version of this paper, which contains a short proof of Fact 3.1 and a proof of Proposition 5.2. A Maple implementation is being written for decomposing $H$-solutions. We shall apply the structure theorem to study the existence of telescopers in the mixed cases in which any two of differential, shift and $q$ shift operators appear.

Acknowledgments. The authors thank Frédéric Chyzak, Bruno Salvy, Michael Singer and anonymous referees for helpful discussions and suggestions.

\section{REFERENCES}

[1] S. A. Abramov. When does Zeilberger's algorithm succeed? Adv. in Appl. Math., 30(3):424-441, 2003.

[2] S. A. Abramov and M. Petkovšek. Proof of a conjecture of Wilf and Zeilberger. Preprints Series of the Inst. Math, Physics and Mechanics, 39(748), Ljubljana, 2001.

[3] S. A. Abramov and M. Petkovšek. Dimensions of solution spaces of $H$-systems. J. Symbolic Comput., 43(5):377-394, 2008.

[4] S. A. Abramov and M. Petkovšek. On the structure of multivariate hypergeometric terms. Adv. in Appl. Math., 29(3):386-411, 2002.

[5] M. Bronstein, Z. Li, and M. Wu. Picard-Vessiot extensions for linear functional systems. In Proc. of ISSAC '05, 68-75, ACM. New York, USA, 2005.

[6] S. Chen, R. Feng, G. Fu and Z. Li. On the structure of compatible rational functions. MM-Res. Preprints, 30: 20-38, 2011.

(http://www.mmrc.iss.ac.cn/pub/mm30/02-Chen.pdf)

[7] S. Chen, F. Chyzak, R. Feng, and Z. Li. The existence of telescopers for hyperexponential-hypergeometric functions. MM-Res. Preprints, 29: 239-267, 2010. (http://www.mmrc.iss.ac.cn/pub/mm29/13-Chen.pdf)

[8] W. Y. C. Chen, Q.-H. Hou, and Y.-P. Mu. Applicability of the $q$-analogue of Zeilberger's algorithm. J. Symbolic Comput., 39(2):155-170, 2005.

[9] C. Christopher. Liouvillian first integrals of second order polynomial differential equations. Electron. J. Differential Equations, 49: 1-7 (electronic), 1999.

[10] R. Feng, M. F. Singer, and M. Wu. An algorithm to compute Liouvillian solutions of prime order linear difference-differential equations. J. Symbolic Comput., 45(3):306-323, 2010

[11] I. Gel'fand, M. Graev, and V. Retakh. General hypergeometric systems of equations and series of hypergeometric type. Uspekhi Mat. Nauk (Russian), Engl. transl. in Russia Math Surveys, 47(4):3-82, 1992.

[12] C. Hardouin and M. F. Singer. Differential Galois theory of linear difference equations. Math. Ann., 342(2):333-377, 2008.

[13] M. Kauers. Algorithms for Nonlinear Higher Order Difference Equations. PhD thesis, RISC-Linz, Linz, Austria, 2005.

[14] G. Labahn and Z. Li. Hyperexponential solutions of finite-rank ideals in orthogonal Ore rings. In Proc. of ISSAC'04, 213-220. ACM, New York, 2004.

[15] Z. Li, M. Wu, and D. Zheng. Testing linear dependence of hyperexponential elements. ACM Commun. Comput. Algebra, 41(1):3-11, 2007.

[16] O. Ore. Sur la forme des fonctions hypergéométriques de plusieurs variables. J. Math. Pures Appl., 9(4):311-326, 1930.

[17] G. H. Payne. Multivariate Hypergeometric Terms. PhD thesis, Penn. State Univ., Pennsylvania, USA, 1997.

[18] M. Sato. Theory of prehomogeneous vector spaces (algebraic part) - the English translation of Sato's lecture from Shintani's note. Nagoya Math. J., 120:1-34, 1990.

[19] M.F. Singer. A note on solutions of first-order linear functional equations. Manuscript for discussions at the Second NCSU-China Symbolic Computation Collaboration Workshop, Hangzhou, March, 2007.

[20] M. van der Put and M.F. Singer. Galois Theory of Difference Equations, volume 1666 of Lecture Notes in Mathematics. Springer-Verlag, Berlin, 1997.

[21] H. Zoladek. The extended monodromy group and Liouvillian first integrals. J. Dynam. Control Systems, 4(1):1-28, 1998. 